\title{
Insulin resistance and systemic metabolic changes in oral glucose tolerance test in 5340 individuals: an interventional study
}

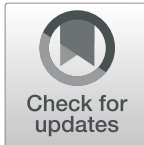

Qin Wang 1,2,3,4* Jari Jokelainen ${ }^{3,5}$, Juha Auvinen ${ }^{3,6}$, Katri Puukka7 , Sirkka Keinänen-Kiukaanniemi ${ }^{3,5,8,9}$, Marjo-Riitta Järvelin ${ }^{3,4,5,10,11}$, Johannes Kettunen ${ }^{1,3,4,12 \dagger}$, Ville-Petteri Mäkinen ${ }^{13,14 \dagger}$ and Mika Ala-Korpela ${ }^{1,2,3,4,15,16,17,18^{*}+}$ (D)

\begin{abstract}
Background: Insulin resistance (IR) is predictive for type 2 diabetes and associated with various metabolic abnormalities in fasting conditions. However, limited data are available on how IR affects metabolic responses in a non-fasting setting, yet this is the state people are mostly exposed to during waking hours in the modern society. Here, we aim to comprehensively characterise the metabolic changes in response to an oral glucose test (OGTT) and assess the associations of these changes with IR.
\end{abstract}

Methods: Blood samples were obtained at 0 (fasting baseline, right before glucose ingestion), 30, 60, and 120 min during the OGTT. Seventy-eight metabolic measures were analysed at each time point for a discovery cohort of 4745 middle-aged Finnish individuals and a replication cohort of 595 senior Finnish participants. We assessed the metabolic changes in response to glucose ingestion (percentage change in relative to fasting baseline) across the four time points and further compared the response profile between five groups with different levels of IR and glucose intolerance. Further, the differences were tested for covariate adjustment, including gender, body mass index, systolic blood pressure, fasting, and 2-h glucose levels. The groups were defined as insulin sensitive with normal glucose (IS-NGT), insulin resistant with normal glucose (IR-NGT), impaired fasting glucose (IFG), impaired glucose tolerance (IGT), and new diabetes (NDM). IS-NGT and IR-NGT were defined as the first and fourth quartile of fasting insulin in NGT individuals.

Results: Glucose ingestion induced multiple metabolic responses, including increased glycolysis intermediates and decreased branched-chain amino acids, ketone bodies, glycerol, and triglycerides. The IR-NGT subgroup showed smaller responses for these measures (mean + 23\%, interquartile 9-34\% at $120 \mathrm{~min}$ ) compared to IS-NGT (34\%, 23-44\%, P< 0.0006 for difference, corrected for multiple testing). Notably, the three groups with glucose abnormality (IFG, IGT, and NDM) showed similar metabolic dysregulations as those of IR-NGT. The difference between the IS-NGT and the other subgroups was largely explained by fasting insulin, but not fasting or $2 \mathrm{~h}$ glucose. The findings were consistent after covariate adjustment and between the discovery and replication cohort.

Conclusions: Insulin-resistant non-diabetic individuals are exposed to a similar adverse postprandial metabolic milieu, and analogous cardiometabolic risk, as those with type 2 diabetes. The wide range of metabolic abnormalities associated with IR highlights the necessity of diabetes diagnostics and clinical care beyond glucose management.

Keywords: Insulin resistance, Metabolic profiling, Oral glucose tolerance test, Impaired glucose tolerance, Impaired fasting glucose, Type 2 diabetes

\footnotetext{
* Correspondence: qin.wang@baker.edu.au; mika.ala-korpela@baker.edu.au

${ }^{\dagger} J$ Cohannes Kettunen, Ville-Petteri Mäkinen, and Mika Ala-Korpela are joint

senior authors.

${ }^{1}$ Computational Medicine, Faculty of Medicine, University of Oulu, Oulu,

Finland

Full list of author information is available at the end of the article
}

(c) The Author(s). 2019 Open Access This article is distributed under the terms of the Creative Commons Attribution 4.0 International License (http://creativecommons.org/licenses/by/4.0/), which permits unrestricted use, distribution, and reproduction in any medium, provided you give appropriate credit to the original author(s) and the source, provide a link to the Creative Commons license, and indicate if changes were made. The Creative Commons Public Domain Dedication waiver (http://creativecommons.org/publicdomain/zero/1.0/) applies to the data made available in this article, unless otherwise stated. 


\section{Background}

Diabetes affects approximately 1 in 11 adults worldwide, and people with diabetes are at a twofold excess risk for cardiovascular disease (CVD) [1, 2]. A decline in insulin sensitivity is an early sign of susceptibility to type 2 diabetes, typically manifested as elevated levels of fasting insulin [3]. Insulin is a key regulator of glucose metabolism by promoting glucose uptake in peripheral tissues and inhibiting glucose production in the liver [4]. Insufficient insulin action results in increased fasting glucose and eventually leads to overt type 2 diabetes [4]. Insulin resistance (IR) is also linked to the development of cardiometabolic complications, the risk arising already prior to the onset of type 2 diabetes $[5,6]$. Studies in the fasting state have identified a cluster of biomarkers robustly associated with IR and predisposing to increased risk for CVD $[3,5,6]$. In the modern society, however, people spend most of their waking hours at a postprandial state, yet we are not aware of epidemiological studies on non-fasting metabolism in representative cohorts.

An oral glucose tolerance test (OGTT) assesses an individual's ability to clear circulating glucose after an ingestion of a 75-g glucose bolus taken after an overnight fast. An OGTT induces a transition from fasting to feeding, and subsequent changes in various metabolic nutrients occur as the body makes adjustments to achieve glucose homeostasis [7]. It is thus feasible to expect that individuals with impaired insulin action are likely to display a widespread systemic abnormality beyond glucose. Although the dynamics of insulin and glucose during an OGTT in both healthy and insulin-resistant individuals are well studied $[8,9]$, much less is known on other, particularly emerging cardiometabolic biomarkers, for example, lipoprotein lipid profiles, amino acids, ketone bodies, and inflammatory markers [10, 11].

Metabolic profiling, simultaneously measuring multiple metabolic measures, has been frequently used in studying metabolic dysregulations in the fasting state. Previous studies have revealed that higher fasting ketone bodies, branched-chain amino acids, and aromatic amino acids are predictive for future type 2 diabetes [10, 12]. Similarly, higher concentration of very-low-density lipoprotein (VLDL) particles and increased triglycerides are associated with higher risk of cardiovascular diseases [13]. In particular, recent genetic studies have suggested that disturbed branched-chain amino acid metabolism and increased triglycerides are on the causal path of cardiometabolic diseases [14, 15]. Metabolic profiling has also been applied to assess the metabolic changes during OGTT in small studies. For example, amino acids, ketone bodies, and triglycerides are decreased during an OGTT and some of these changes seem to be blunted in obese and insulin-resistant individuals [7, 16-21]. However, all these studies have been limited in their sample size (up to a few hundred individuals) and often spanned only two time points (pre- and post-OGTT).

In this study, we performed an OGTT across 4 time points and quantified 78 metabolic measures for a total of 5340 individuals (over 21,000 serum samples) from 2 independent population-based cohorts. Our aims were (1) to comprehensively characterise systemic metabolic responses to oral glucose in large scale and (2) to investigate how insulin resistance is associated with postprandial metabolic dysregulation across multiple clinical categories of glucose intolerance. To our knowledge, this is the first population-based large-scale metabolomics time-series study of an OGTT, providing new insights into the metabolic consequences of insulin resistance in non-fasting conditions.

\section{Methods}

\section{Study population}

The Northern Finland Birth Cohort 1966 (NFBC66) was initiated to study factors affecting preterm birth and subsequent morbidity in the two northernmost provinces in Finland [22]. It included 12,058 children born alive, comprising 96\% of all births during 1966 in the region. The participants were further followed up at the age of 1, 14, 31, and 46 years. Data collection conducted in 2012 at their age of 46, including clinical examination and serum sampling, was available for 5839 individuals. Among them, 4745 study participants, who were free of prior diagnosed diabetes, underwent metabolic profiling of OGTT serum samples ( $97 \%$ had 4 time points), and had information on baseline fasting insulin and glucose, were used in this study.

The Oulu1945 cohort studies ageing populations in Oulu, Finland. It was started in 2000 and was originally comprised of 1400 individuals born in 1945. In the follow-up study conducted in 2015, data collection including clinical examination and serum sampling was available for 717 participants. Among them, 595 participants who were free of prior diagnosed diabetes, underwent metabolic profiling of OGTT samples (92\% had 4 time points), and had data on baseline fasting insulin and glucose were included.

\section{Clinical assessment}

Subjects underwent a 2-h, 75-g OGTT after an overnight fasting. Blood samples were obtained at 0 (fasting baseline, right before glucose ingestion), 30, 60, and 120 min during the OGTT. Plasma glucose were analysed by an enzymatic dehydrogenase method (Advia 1800, Siemens Healthcare Diagnostics, Tarrytown, NY, USA) and serum insulin by a chemiluminometric immunoassay (Advia Centaur XP, Siemens Healthcare Diagnostics, Tarrytown, NY, USA). Insulin resistance was estimated by fasting insulin, homeostasis model assessment of 
insulin resistance (HOMA-IR), and insulin sensitivity index-Matsuda (ISI-Matsuda). First-phase insulin secretion, an index of beta-cell function, was measured by insulinogenic index. The formulas for these models are shown in the legend for Table 1.

According to an individual's insulin resistance status and the American Diabetes association 2003 criteria [8], participants were classified into five groups (Table 1, Fig. 1, and Additional file 1: Table S1):

1) Insulin-sensitive subgroup of normal glucose tolerance (IS-NGT, fasting insulin at the bottom quartile of NGT and fasting glucose $<5.6 \mathrm{mmol} / \mathrm{L}$ and 2-h glucose $<7.8 \mathrm{mmol} / \mathrm{L}$ )

2) Insulin-resistant subgroup of normal glucose tolerance (IR-NGT, fasting insulin at the top quartile of NGT and fasting glucose $<5.6 \mathrm{mmol} / \mathrm{L}$ and 2-h glucose $<7.8 \mathrm{mmol} / \mathrm{L}$ )

3) Impaired fasting glucose (IFG, fasting glucose between 5.6 and $6.9 \mathrm{mmol} / \mathrm{L}$ and 2-h glucose $<7.8$ $\mathrm{mmol} / \mathrm{L}$ )

4) Impaired glucose tolerance (IGT, fasting glucose $\leq$ $6.9 \mathrm{mmol} / \mathrm{L}$ and 2-h glucose between 7.8 and 11.0 $\mathrm{mmol} / \mathrm{L}$ )

5) New onset of type 2 diabetes (NDM, fasting glucose $\geq 7.0 \mathrm{mmol} / \mathrm{L}$ or 2 -h glucose $\geq 11.1 \mathrm{mmol} / \mathrm{L}$ )

\section{Metabolic profiling}

The human serum metabolome is dominated by hydrophobic lipid-like molecules, including diglycerides, triglycerides, phospholipids, fatty acids, steroids, and steroid derivatives [23]. These lipids are packed in various lipoprotein particles, e.g. VLDL, intermediatedensity lipoprotein (IDL), low-density lipoprotein (LDL), and high-density lipoprotein (HDL). Other metabolites found in high abundance in serum include amino acids, glucose, lactate, and several waste or catabolic by-products, such as urea and creatinine [23]. Here, a nuclear magnetic resonance (NMR) spectroscopy metabolomics platform was used to measure all the detectable lipids and metabolites in a nonselective way. The high-throughput NMR metabolomics platform was applied to quantify over 200 lipid and metabolite measures from serum samples collected at $0,30,60$, and 120 min during an OGTT challenge. The platform applies a single experimental setup, which allows for simultaneous quantification of standard clinical lipids, 14 lipoprotein subclasses, and individual lipids (triglycerides, phospholipids, free and esterified cholesterol) transported by these particles, multiple fatty acids, glucose and various glycolysis precursors, ketone bodies, and amino acids in absolute concentration units [24-26]. As the total lipids and

Table 1 Characteristics of the Northern Finland Birth Cohort 1966

\begin{tabular}{|c|c|c|c|c|c|c|}
\hline & \multicolumn{3}{|c|}{ Without glucose abnormality } & \multicolumn{3}{|c|}{ With glucose abnormality } \\
\hline & All NGT & IS-NGT & IR-NGT & IFG & IGT & NDM \\
\hline N & 2847 & 708 & 713 & 1380 & 412 & 106 \\
\hline Age [year] & $46.7[46.2-47.1]$ & $46.5[46.2-46.9]$ & $46.8[46.4-47.3]$ & $46.6[46.2-47.1]$ & $46.7[46.3-47.2]$ & $46.8[46.3-47.2]$ \\
\hline Men [\%] & 34 & 33 & 38 & 61 & 48 & 63 \\
\hline BMI $\left[\mathrm{kg} / \mathrm{m}^{2}\right]$ & $24.9[22.7-27.6]$ & $23.1[21.6-25.1]$ & $27.8[25.1-30.9]$ & $27.0[24.6-29.9]$ & $29.2[26.3-32.4]$ & $30.0[27.3-33.3]$ \\
\hline Systolic blood pressure [mmHg] & 120 [111-131] & 118 [109-128] & 125 [114-135] & 128 [118-138] & 130 [122-142] & 136 [124-150] \\
\hline Diastolic blood pressure [mmHg] & 82 [75-89] & 79 [73-86] & 85 [78-93] & 86 [80-93] & 90 [82-97] & 94 [86-100] \\
\hline Triglycerides [mmol/L] & $1.0[0.7-1.3]$ & $0.8[0.6-1.1]$ & $1.3[1.0-1.7]$ & $1.3[0.9-1.8]$ & $1.6[1.1-2.2]$ & $1.7[1.3-2.2]$ \\
\hline LDL cholesterol [mmol/L] & $1.8[1.5-2.2]$ & $1.7[1.5-2.2]$ & $1.9[1.6-2.4]$ & $2.0[1.6-2.4]$ & 1.9 [1.6-2.3] & $2.0[1.7-2.3]$ \\
\hline HDL cholesterol [mmol/L] & $1.7[1.4-1.9]$ & $1.8[1.6-2.0]$ & $1.5[1.3-1.7]$ & $1.5[1.3-1.8]$ & $1.4[1.2-1.7]$ & $1.4[1.1-1.6]$ \\
\hline Fasting insulin [mU/L] & $6.5[4.6-9.5]$ & $3.6[3.0-4.1]$ & $12.2[10.6-14.9]$ & $9.8[6.9-13.5]$ & $12.4[8.3-18.3]$ & $15.5[10.5-24.4]$ \\
\hline $2 \mathrm{~h}$ insulin [mU/L] & $37.0[25.2-53.2]$ & $25.2[17.9-34.2]$ & $59.9[41.9-92.8]$ & $47.9[31.6-77.6]$ & $120.4[68.8-187.4]$ & $129.9[66.0-195.5]$ \\
\hline Fasting glucose $[\mathrm{mmol} / \mathrm{L}]$ & $5.2[5.0-5.4]$ & $5.0[4.8-5.3]$ & $5.3[5.1-5.4]$ & $5.8[5.7-6.0]$ & $5.7[5.4-6.1]$ & $6.9[6.3-7.2]$ \\
\hline $2 \mathrm{~h}$ glucose $[\mathrm{mmol} / \mathrm{L}]$ & $5.2[4.6-5.9]$ & $4.9[4.3-5.6]$ & $5.6[4.9-6.3]$ & $5.9[5.1-6.6]$ & $8.6[8.1-9.2]$ & $11.5[8.4-12.6]$ \\
\hline HOMA-IR & $1.5[1.0-2.2]$ & $0.8[0.7-0.9]$ & $2.8[2.5-3.5]$ & $2.5[1.8-3.5]$ & $3.2[2.0-4.7]$ & $4.5[3.2-7.6]$ \\
\hline ISI (Matsuda) & $6.3[4.4-8.6]$ & $10.6[8.9-12.7]$ & $3.4[2.6-4.2]$ & $3.8[2.6-5.5]$ & $2.5[1.7-3.9]$ & $1.9[1.2-3.0]$ \\
\hline Insulinogenic index & $21.7[13.3-38.2]$ & $15.7[9.9-26.2]$ & $32.2[20.2-49.2]$ & $20.7[12.6-33.2]$ & $18.0[10.5-30.2]$ & $10.8[7.6-20.2]$ \\
\hline
\end{tabular}

Values are median [interquartile range]. Characteristics for the replication cohort Oulu45 is shown in Additional file 1: Table S1

Abbreviations: BMI body mass index, NGT normal glucose tolerance, IFG isolated impaired fasting glucose, IGT isolated impaired glucose tolerance, NDM new type 2 diabetes, IS-NGT insulin-sensitive individuals within NGT (at the first quartile of fasting insulin within NGT), IR-NGT insulin-resistant individuals within NGT (at the top quartile of fasting insulin within NGT)

HOMA-IR = fasting glucose $(\mathrm{mmol} / \mathrm{L}) \times$ fasting insulin $(\mathrm{mlU} / \mathrm{L}) / 22.5$

ISI-Matsuda $=10,000 /$ square root of [fasting glucose $(\mathrm{mg} / \mathrm{dL}) \times$ fasting insulin $(\mathrm{mlU} / \mathrm{L}) \times$ mean glucose $\times$ mean insulin during OGTT]

Insulinogenic index $=\left(\right.$ Insulin $_{30\left(\mathrm{mIU}_{\mathrm{L}}\right)}-$ Insulin $\left._{0}\right) /\left(\mathrm{Glucose}_{30\left(\mathrm{mmol}_{\mathrm{L}}\right)}-\right.$ Glucose $\left._{0}\right)$ during OGTT 


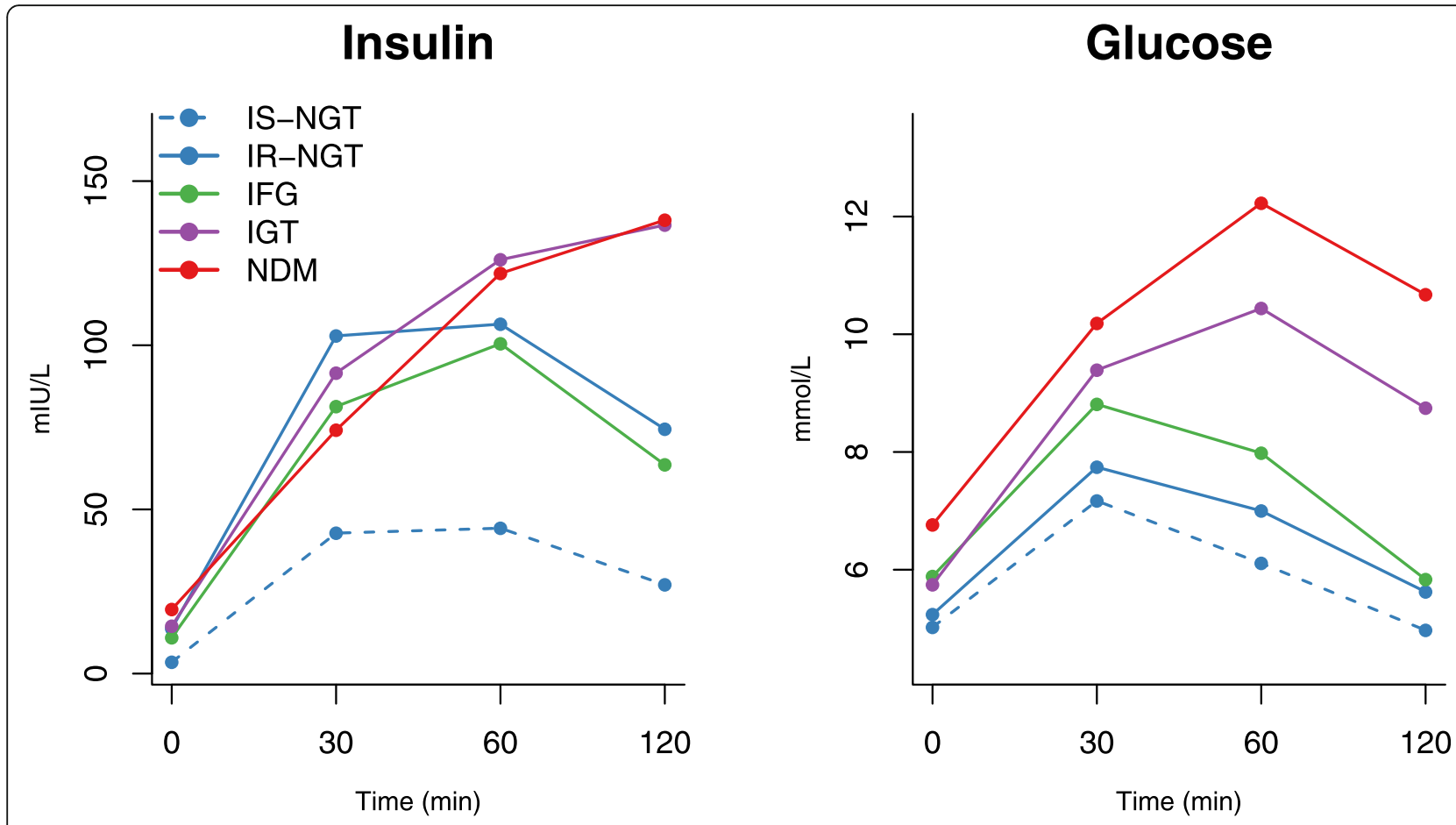

Fig. 1 Mean concentration of insulin and glucose at 0, 30,60, and 120 min during an oral glucose tolerance test. Insulin and glucose trajectories for insulin-sensitive subgroup of normal glucose tolerance (IS-NGT, dashed blue, $n=708$ ), insulin-resistant subgroup of normal glucose tolerance (IR-NGT, solid blue, $n=713$ ), impaired fasting glucose (IFG, green, $n=1380$ ), impaired glucose tolerance (purple, $n=412$ ), and newly-diagnosed type 2 diabetes (red, NDM, $n=106$ ) are shown. IS-NGT was defined as the bottom quartile of fasting insulin within NGT, and IR-NGT was defined as the top quartile. The dots denote mean absolute concentrations. Interquartile ranges are listed in Table 1

individual lipids within the same lipoprotein subclass are highly correlated [27], we chose a priori to analyse the total lipids in the 14 subclasses and limit specific lipids for the 4 major fractions (VLDL, IDL, LDL, and HDL). These together with all the fatty acids and nonlipid measures provided by this platform, in total 77 measures, were used in the present study. A similar metabolic panel has been widely applied in previous studies $[3,28,29]$.

\section{Statistical analyses}

In total, 78 metabolic measures were used in the analyses. Of those, 77 were measured by NMR metabolomics and glucose by a clinical assay. Insulin was treated as an exposure in this study. All analyses were undertaken in the $\mathrm{R}$ programming environment (version 3.5.1). Primary analyses were conducted using NFBC66, and key results were replicated in Oulu1945.

To study the physiological response to an OGTT, metabolic trajectories for NGT individuals were reported. Metabolic trajectories were calculated as percentage changes in relative to baseline concentration at 30, 60, and $120 \mathrm{~min}$, respectively, e.g. (Concentration $_{120 \mathrm{~m}}$ - Concentration $\left.{ }_{0 \mathrm{~m}}\right) /$ Concentration $_{0 \mathrm{~m}} \times 100 \%$. In the formula, metabolic concentrations are in their original units, e.g. $\mathrm{mmol} / \mathrm{L}$. The significance of a change was evaluated via paired $t$ test by comparing the metabolite concentration at post-load time points against the fasting baseline. The analyses were repeated for men and women separately. Due to the correlated nature of the metabolic measures, 19 principle components were able to explain $95 \%$ variation of the 78 measures; therefore, $P<0.05 / 19 / 4=0.0006$ was considered statistically significant after correcting for multiple comparisons (corrected for 19 independent components and across 4 time points) [30, 31].

To assess whether metabolic trajectories would be different across the groups, two-way ANOVA was used, with metabolite change (\%) as the response, time points $\times$ groups as the interaction term, and gender as the covariate. In total, 60 out of 78 measures showed significant interaction of time points and groups, suggesting the metabolic trajectories would be different between the groups for these measures (Additional file 2: Table S2). $t$ tests were further used to compare the metabolic trajectories between IR-NGT and IS-NGT across the 78 measures. For those metabolic measures that showed significant differences between IR-NGT and IS-NGT, we further assessed their differences between IR-NGT and those with IGT or NDM. 
In addition, sensitivity analyses were conducted to assess the effect of potential covariates for those measures that showed significant differences between IR-NGT and IS-NGT. Linear regression models were used to quantify the metabolic differences between the groups, using 2-h change in metabolite concentration as the response variable and group category as the independent variable. Four sets of covariates were used: (1) sex, (2) sex + BMI + systolic blood pressure, (3) sex + baseline glucose +2 -h glucose, and (4) sex + fasting insulin. Metabolite concentrations at baseline and $2 \mathrm{~h}$ were log-transformed, and the changes between the baseline and $2 \mathrm{~h}$ were scaled to baseline SD.

\section{Results}

Two population cohorts were used to study the metabolic changes during an OGTT. The primary analyses were conducted in 4745 individuals in the NFBC66 (mean age 47 years, $44 \%$ men, Table 1), and the key results were replicated in 595 participants in the Oulu1945 (mean age 69 years, $41 \%$ men, Additional file 1: Table S1). Among the participants in the NFBC66, $60 \%$ of individuals had normal fasting and 2-h glucose (NGT), $29 \%$ had impaired fasting glucose (IFG), and $11 \%$ had impaired 2-h glucose tolerance (IGT or NDM). Although NGT individuals are generally considered healthy, the IR-NGT subgroup had over 3 times higher fasting insulin than the IS-NGT. After glucose ingestion, these insulin-resistant individuals secreted even more insulin in the early phase $(30 \mathrm{~min})$, yet they were still unable to restore glucose levels back to the pre-OGTT levels after $2 \mathrm{~h}$ (Table 1 and Fig. 1). The IR-NGT individuals were also more likely to be male and had higher BMI, blood pressure, and fasting triglycerides and lower HDL cholesterol (Table 1). Similar characteristics were observed for IFG, IGT, and NDM, and their fasting insulin levels were comparable to IR-NGT, ranging from 2.7 to 4.3 times more than IS-NGT.

\section{Metabolic trajectories under normal glucose tolerance}

Selected responses to an OGTT for the NGT individuals are summarised in Fig. $2(P<0.0006$ at any time point), and results for all measures are available in Additional file 1: Figure S1 and Additional file 2: Table S3. During the OGTT, glycolysis-related metabolic measures (pyruvate and lactate) were primarily increased during 30 and $60 \mathrm{~min}$ (peaking at $60 \mathrm{~min}$ with $49 \%$ [interquartile $19 \%, 74 \%$ ] and $31 \%$ [14\%, 47\%], respectively), lagging approximately $30 \mathrm{~min}$ behind the glucose rise (Fig. 2a). A smaller increase was seen with citrate (peaking with $7 \%[-2 \%, 15 \%]$ at 30 min). On the other hand, ketone bodies betahydroxybutyrate and acetoacetate were continuously reduced after glucose ingestion and lowered by $26 \%$ $(8 \%, 42 \%)$ and $41 \%(29 \%, 56 \%)$ at $120 \mathrm{~min}$, respectively. Similarly, almost all amino acids were decreased during the OGTT, except for alanine (Fig. 2b). Branched-chain (isoleucine, leucine, and valine) and aromatic amino acids (phenylalanine and tyrosine) were decreased (15 to $45 \%)$ more than the other amino acids (6 to 10\%) at $120 \mathrm{~min}$. Acetate and glycerol were decreased throughout the OGTT and reduced by $24 \%(16 \%, 33 \%)$ and $39 \%(25 \%, 55 \%)$ at $120 \mathrm{~min}$, respectively (Fig. 2c). Changes in lipids and fatty acids were generally smaller in comparison to the aforementioned non-lipid measures (Fig. 2 and Additional file 1: Figure S1A). The largest changes in lipids were seen for the total lipids in extremely large, very large, large, and medium VLDL particles, with 11 to $32 \%$ reduction at $120 \mathrm{~min}$, after initial increases at $60 \mathrm{~min}$ (e.g. large VLDL in Fig. 2c). All HDL subclass measures were suppressed during the OGTT, with a 2 to $9 \%$ decrease at $120 \mathrm{~min}$ (e.g. very large HDL in Fig. 2c). Interestingly, circulating triglycerides in all main lipoprotein particles, VLDL, IDL, LDL, and HDL, were decreased at $120 \mathrm{~min}$ (1 to $11 \%$, e.g. VLDL-TG and HDL-TG in Fig. 2c). Inconsistent and small changes were seen in the corresponding cholesterol concentrations (see Additional file 1: Figure S1A for details).

\section{Metabolic trajectories under insulin resistance}

Metabolic trajectories of IR-NGT were compared to those of IS-NGT (Fig. 3). The analyses were restricted to individuals with normal glucose tolerance to rule out any secondary effects from hyperglycaemia. Full results for all 78 measures are available in Additional file 1: Figure S2 and Additional file 2: Table S4. Pronounced differences were observed in multiple metabolic pathways including glycolysisrelated metabolites, branched-chain amino acids, ketone bodies, and triglyceride-related measures (Fig. 3b-e). Typical differences were initially small at $30 \mathrm{~min}$ and became more pronounced from $60 \mathrm{~min}$ onwards (except for insulin and glucose). At $120 \mathrm{~min}$, the IR-NGT individuals showed higher increase in glucose yet smaller increase in pyruvate, lactate, and alanine levels. Also, they displayed smaller decrease in branched-chain amino acids and ketone bodies as well as triglyceride-related measures. Overall, the changes at 120 min across these measures (Fig. 3b-e) were 34\% (interquartile $23-44 \%)$ in IS-NGT, whereas only $23 \%(9-34 \%)$ in IRNGT. These differences were statistically significant with $P<0.0006$ (Additional file 1: Figure S2B). The results were consistent when stratified by sex (Additional file 1: Figure S3). Also, the results were similar when we compared the top and bottom quartiles of the HOMA-IR and 1/Matsuda indices (Additional file 1: Figure S4). 

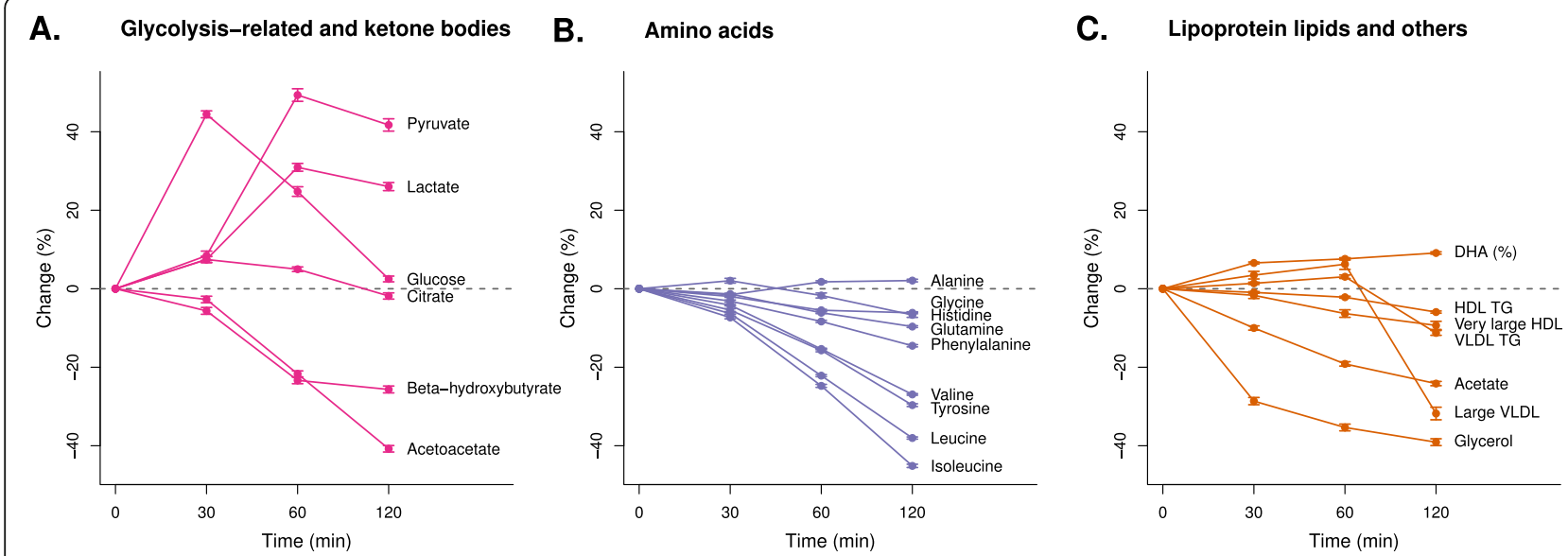

Fig. 2 Selected metabolic changes in response to an oral glucose tolerance test in individuals with normal glucose tolerance. The dots and error bars denote mean percent change and $95 \% \mathrm{Cl}$. Percent change is defined as the absolute change in relative to baseline. a Glycolysis-related and ketone bodies. b Amino acids. c Lipoprotein lipids and others

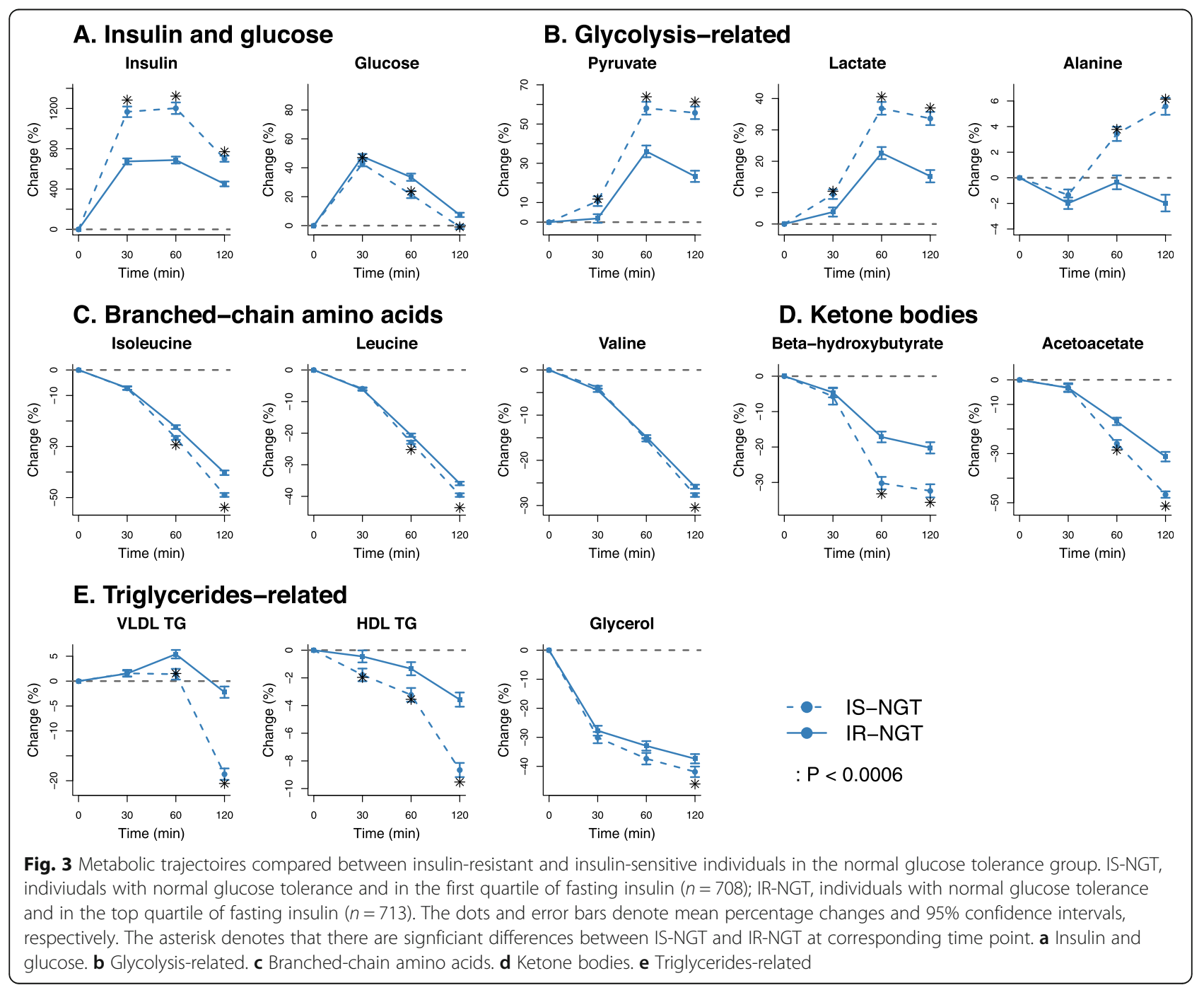


Metabolic trajectories under prediabetes and diabetes

Figure 4 (Additional file 2: Table S5) presents the comparison of the metabolic trajectories in individuals with 2-h impaired glucose tolerance (IGT or NDM) and those of IR-NGT. Although large differences in glucose responses were observed by definition, these two groups showed marginal differences in metabolic responses in glycolysis products, branched-chain amino acids, ketone bodies, and triglyceride-related measures (Fig. 4b-e). In addition, the IFG individuals who had normal 2-h glucose response but high fasting glucose (5.9 vs $5.2 \mathrm{mmol} /$ L in IFG and IR-NGT) also showed marginal differences in metabolic trajectories compared to those of IR-NGT (Additional file 1: Figure S5). The metabolic trajectories in percent change and absolute concentrations across all five individual groups (IS-NGT, IR-NGT, IFG, IGT, and NDM) are shown in Additional file 1: Figures S6 and S7. Results corresponding to those shown in Figs. 2, 3, and 4 for the discovery cohort NFBC66 are replicated in the
Oulu1945 cohort (see Additional file 1: Figure S8 for detailed results).

\section{Metabolic responses associated with IR with or without glucose abnormality}

Figure 5a displays the distributions of insulin resistance measured by HOMA-IR and Matsuda index in individuals with IS-NGT, IR-NGT, and IFG + IGT + NDM. Despite the IFG + IGT + NDM group having impaired glucose metabolism by definition, these individuals together with the IRNGT group showed comparable HOMA-IR and Matsuda indices. Interestingly, these two groups also showed similar differences in the 2-h metabolite responses when compared to the IS-NGT group (Fig. 5b). This was consistently observed in the two independent cohorts. The metabolic differences associated with IR-NGT and IFG + IGT + IGT remained the same or became slightly attenuated after adjusting for BMI, systolic blood pressure, baseline glucose, and 2-h glucose (Fig. 6). By contrast, the associations were

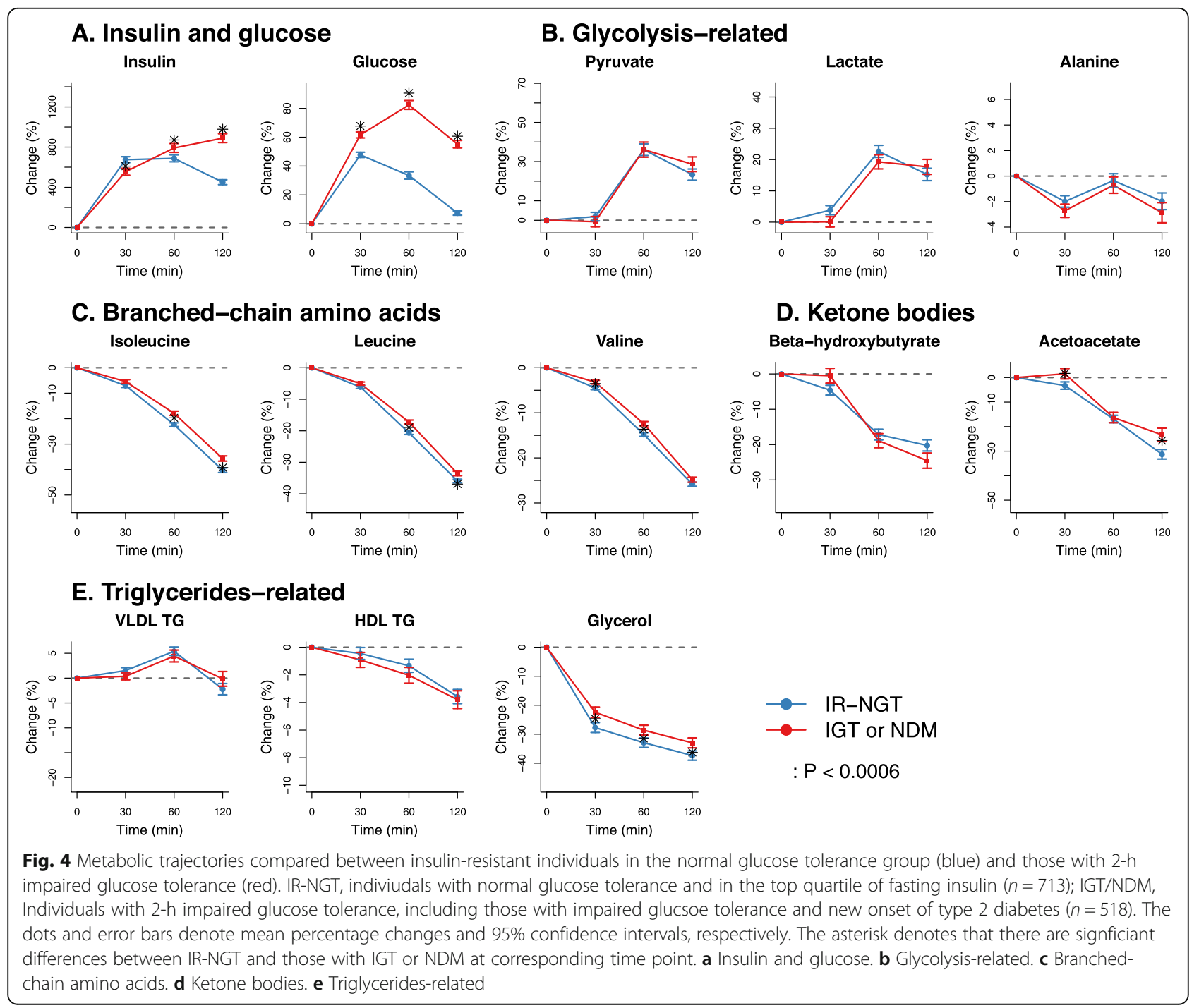




\section{A. Comparing IR in NGT and IFG+IGT+NDM}

HOMA-IR

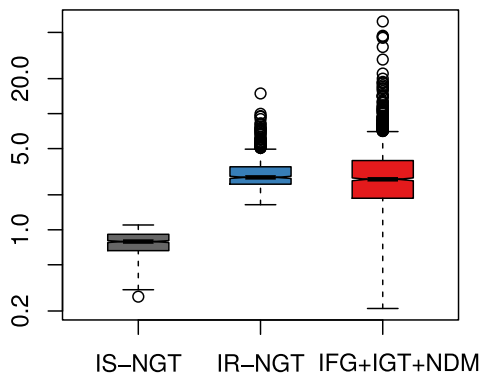

Matsuda-ISI

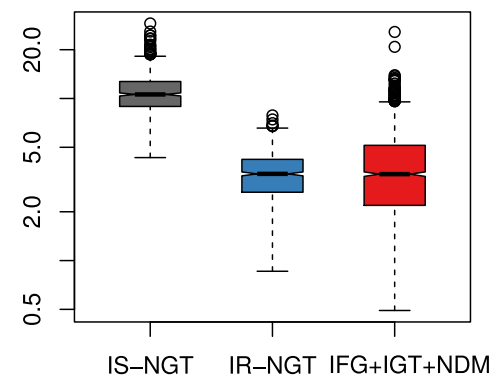

\section{B. Comparing metabolic associations of IR with or without glucose abnormality}

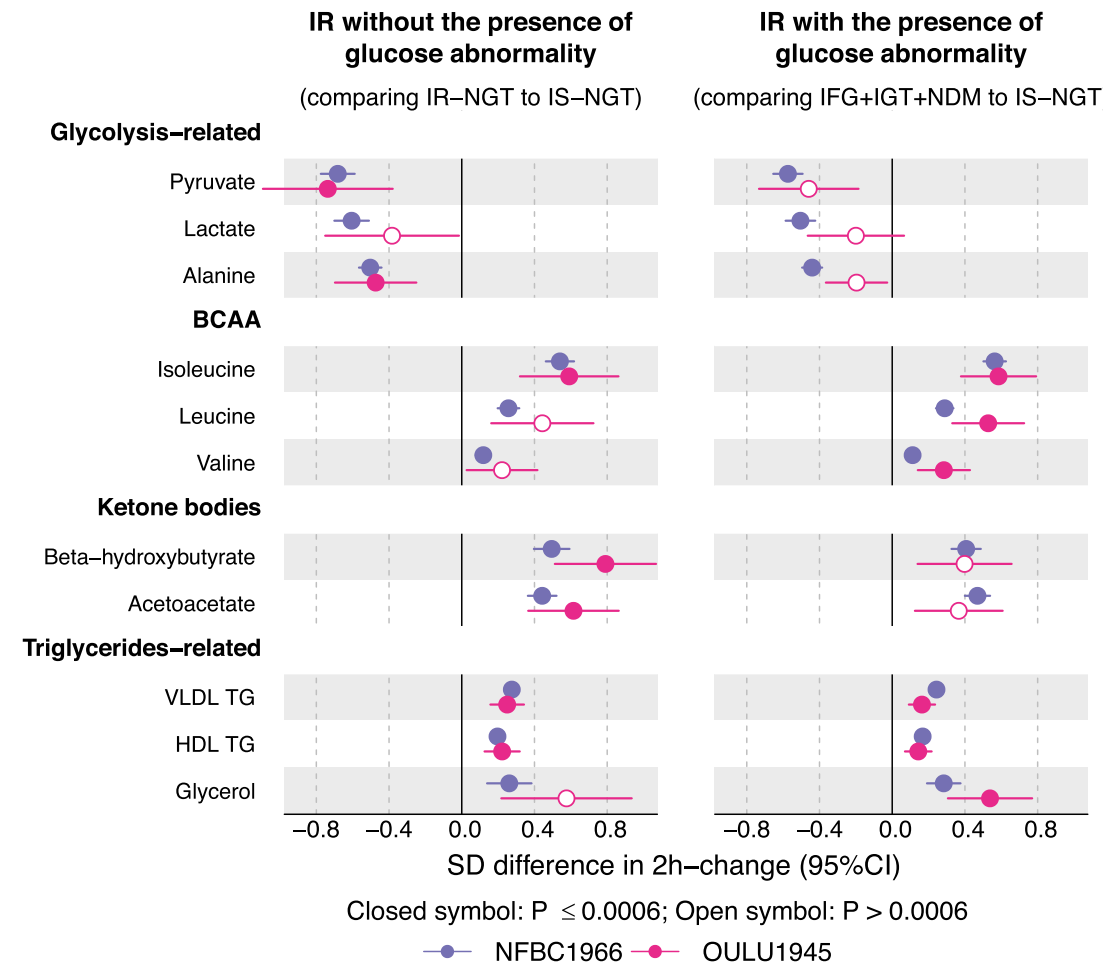

Fig. 5 Summary and replication. a Estimated insulin resistance in IS-NGT (grey), IR-NGT (blue), and pooled of IFG, IGT, and NDM (red) in NFBC66. b Two-hour metabolic responses associated with IR with or without glucose abnormality in NFBC66 (purple) and replicated in Oulu45 (red). Groups were compared by linear regression models with the 2 -h concentration change as the response variable. Baseline and 2-h metabolite concentrations were log-transformed, and the changes between 2-h and baseline metabolite concentrations were scaled to baseline SD. Group sizes within NFBC66: $n=708$ in IS-NGT, $n=713$ in IR-NGT, and $n=1898$ in combined IFG, IGT, and NDM. Group sizes within Oulu1945: $n=62$ in IS-NGT, $n=64$ in IR-NGT, and $n=343$ in combined IFG, IGT, and NDM

substantially attenuated to almost null after adjusting for fasting insulin. Similar results were observed when IFG, IGT, and NDM were individually compared to IS-NGT with the adjustments (Additional file 1: Figure S9).

Lastly, we observed distinctive patterns in fasting metabolic concentrations and the 2-h metabolite responses (Additional file 1: Figures S7 and S10).
Branched-chain amino acids and triglycerides in IR individuals were higher at baseline and exhibited less decrease at $2 \mathrm{~h}$, compared to the IS-NGT group. Glycolysis-related measures were higher in IR individuals at baseline, but increased less at $2 \mathrm{~h}$, whereas ketone bodies seemed to be lower at baseline, but decreased less at $2 \mathrm{~h}$ compared to the IS-NGT group. 


\section{A. IR without glucose abnormality}

(IR-NGT vs IS-NGT)

Glycolysis-related

Pyruvate

Lactate

Alanine

BCAA

Isoleucine

Leucine

Valine

\section{Ketone bodies}

Beta-hydroxybutyrate
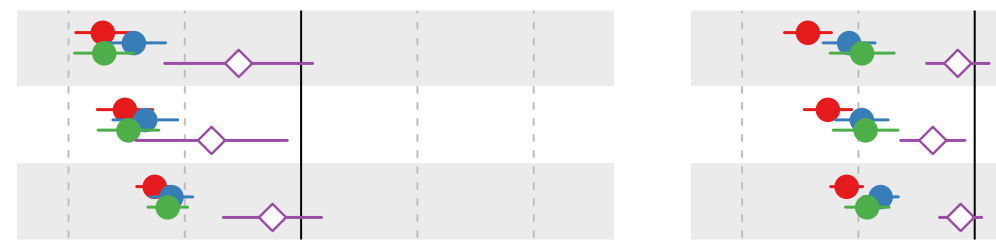

B. IR with glucose abnormality (IFG+IGT+NDM vs IS-NGT)

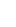
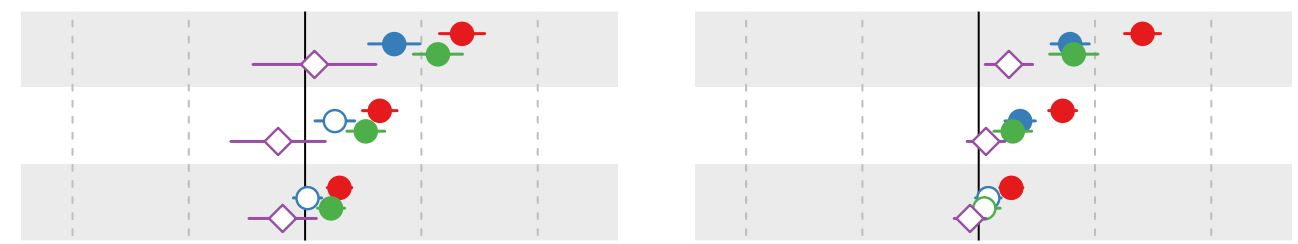

\section{Acetoacetate}

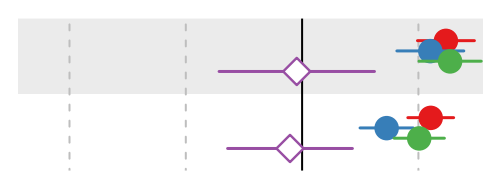

Triglycerides-related
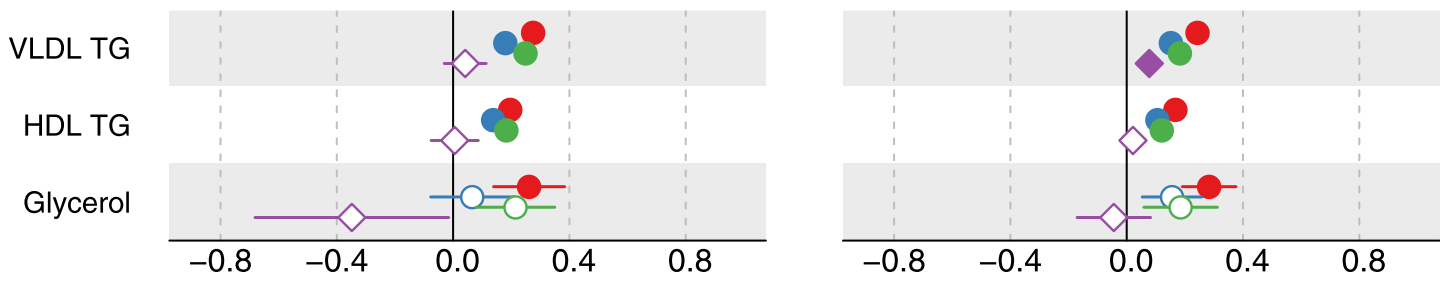

SD difference in $2 \mathrm{~h}$-change $(95 \% \mathrm{Cl})$

Closed symbol: $P \leq 0.0006$; Open symbol: $P>0.0006$

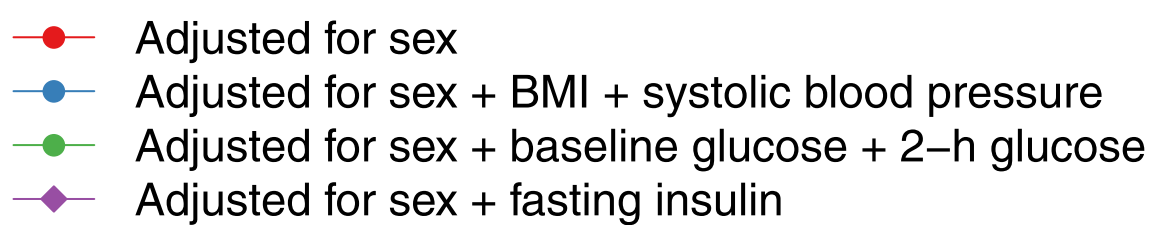

Fig. 6 Group comparison adjusted for baseline factors in the NFBC66 cohort. a Differences in 2-h changes between the IR-NGT ( $n=713$ ) and the IS-NGT group $(n=708)$. b Differences in 2-h changes in the combined IFG, IGT, and NDM $(n=1898)$ and the IS-NGT group $(n=708)$. Groups were compared by linear regression models with the 2 -h concentration change as the response variable. Baseline and 2 -h metabolite concentrations were log-transformed, and the changes between 2-h and baseline metabolite concentrations were scaled to baseline SD. Insulin was log-transformed

\section{Discussion}

We profiled four time points of OGTT data for in total 5340 Finnish individuals from 2 independent cohorts to obtain new large-scale population-based information on how insulin resistance is associated with a systemic post-load metabolic dysregulation. These changes include adverse modifications in multiple cardiometabolic biomarkers suggesting that insulin resistance may underlie the shared susceptibility to diabetes and CVD also in the post-load milieu. Our study is important because most people spend a significant amount of their daily lives in a postprandial state-this aspect of insulin resistance has not been captured in previous metabolomics studies of fasting samples. The 
results also carry practical significance: we found that IR-associated metabolic aberrations exist already in participants with normal glucose tolerance (with implications for CVD risk) and are similar in extent to those observed in type 2 diabetes.

The large sample size and multiple metabolomics time points allowed us to obtain accurate and systemic understanding of the expected metabolic changes in response to glucose ingestion in people with normal glucose tolerance. Our temporal data on the 2-h changes were consistent with previous small studies with pre- and post-OGTT measures and support the known action of insulin in promoting glycolysis metabolism (pyruvate and lactate) and suppression of ketogenesis (ketone bodies), proteolysis (amino acids), and lipolysis (glycerol) $[4,7,18,20]$. Additionally, our results showed that glucose ingestion also reduces the circulating concentration of triglycerides in VLDL particles after the initial increase during the first $60 \mathrm{~min}$. This may reflect a complex balance of hepatic triglyceride production between increased conversion from excess glucose and reduced reesterification from free fatty acids (as a result of reduced lipolysis) [4]. A general observation is that different metabolic pathways were differentially affected. For example, concentrations of glycolysis-related measures peaked within $2 \mathrm{~h}$, whilst most other measures (e.g. amino acids, ketone bodies, and triglycerides) continuously decreased during the $2 \mathrm{~h}$ and had an evident trend afterwards.

The extensive metabolic data demonstrate that insulinresistant individuals had systematically smaller relative metabolic responses in comparison to the insulin-sensitive ones. Some of these blunted changes have been previously reported for insulin-resistant or obese individuals separately in small studies, e.g. for lactate $[7,20]$, beta-hydroxybutyrate $[7,20]$, isoleucine [7, 20], glycerol [7], and VLDL-TG [16, 18]. Interestingly, the metabolic measures which showed blunted changes in insulin-resistant individuals in this study have been also associated with insulin resistance in the fasting state [28]. It has been suggested that insulin resistance is associated with higher fasting glycolysis-related measures and greater fasting concentrations of branched-chain amino acids, glycerol, and triglycerides [28]. Prospective studies have suggested that the associated metabolic dysregulations at fasting state are predictive of future cardiometabolic risk $[10,11,29,32]$. Further, recent Mendelian randomisation analyses have indicated a causal link from insulin resistance to higher branched-chain amino acids and triglycerides in the fasting state [3]. Our results here underline the possibility that fasting concentrations may also reflect the insufficient suppression of branched-chain amino acids and triglycerides in the postprandial state in the insulin-resistant individuals. Regardless of the exact sequence of events, this study provides new evidence that insulin-resistant individuals are at greater cardiometabolic risk both in the fasting and post-load settings.
The comparison between IR-NGT and IS-NGT addressed the differences in IR whilst having normal glucose metabolism. We also performed a mirror experiment where we compared the metabolic trajectories of IFG, IGT, and NDM to IR-NGT (varying glucose levels but minimising the differences in IR). Interestingly, we found similar metabolic dysregulations in individuals with prediabetes and diabetes to those of insulin-resistant individuals with normal glucose metabolism. These findings suggest limited impact of glucose on these metabolic associations. This interpretation is reinforced by our adjusted analyses: the metabolic dysregulations appear to be exclusively driven by insulin resistance but not fasting or 2-h glucose. Type 2 diabetes, characterised by increased circulating glucose concentrations, is a known risk factor for CVD. However, a meta-analysis of prospective studies found only a marginal association between circulating glucose and CVD outcomes [2]. Consistently, a metaanalysis of over 300 trials found limited evidence to support glucose-lowering drugs would reduce the risk of cardiovascular disease and all-cause mortality in patients of established type 2 diabetes [33]. By contrast, individuals at the stage of IR-NGT or prediabetes are reported to have higher risk of CVD [6, 34]. Taking these together, it seems that long-term exposure for the metabolic consequences of insulin resistance across multiple tissues would account for the concerting development of type 2 diabetes and cardiometabolic complications $[5,6]$. Our study revealed that glucoseindependent postprandial dysfunction might be a novel component of this exposure that is hitherto poorly recognised as a potential interventional target.

Large-scale population studies and multiple time points of metabolomics data gave us a unique opportunity to study the systemic metabolic trajectories across multiple clinical glucose categories. Analyses with multiple testing, multivariate adjustments, and replication in an independent cohort all point towards the robustness of the current findings. The associations of insulin resistance with the metabolic changes were consistent when assessed across three different surrogate markers of insulin resistance. However, we acknowledge that insulin resistance markers may reflect a composite state of insulin sensitivity levels of multiple tissues. In order to understand the metabolic signatures of specific tissues, further experiments are required. In addition, the results were coherent whether the metabolic changes were assessed via relative or absolute concentration changes. The associations remained similar between men and women, between middle-aged and older individuals, and also between those with or without the presence of glucose abnormality. However, ethnic and socioeconomic context should be taken into account when extending these results to other populations. The OGTT corresponds to the ingestion of sugary drinks, but not mixed meals, and thus, these results should not be generalised to post-meal metabolic responses. 


\section{Conclusions}

In conclusion, our results highlight the detrimental effects of insulin resistance on systemic metabolism after glucose ingestion. The population health impact of these metabolic consequences is likely substantial given the spurious and energy-dense eating patterns in the modern world, i.e. people are mostly living in a non-fasting state and consume high amounts of added sugar and refined carbohydrates. The observed metabolic effects manifest very early on, and these findings suggest new avenues to understand the increased CVD risk in insulin resistance and diabetes. It might therefore be beneficial if diabetes diagnostics and clinical care would be extended beyond glucose management. We call for better recognition of postprandial dysfunction beyond glucose tolerance categories as an important cardiometabolic risk factor, and new preventive efforts and strategies to reverse all aspects of metabolic dysregulation. We maintain that this is particularly important at the early stages of insulin resistance, and may also hold untapped therapeutic opportunities.

\section{Supplementary information}

Supplementary information accompanies this paper at https://doi.org/10. 1186/s12916-019-1440-4.

Additional file 1: Table S1. Characteristics of the Oulu1945 cohort. Figure S1. A. Changes in 78 metabolic measures in response to an oral glucose tolerance test. B. Corresponding significance levels for A. Figure S2. A. Difference in metabolic changes between IR-NGT and IS-NGT. B. Corresponding significance level for A. Figure S3. Differences in metabolic changes associated with insulin resistance stratified by sex. Figure S4. Differences in metabolic changes associated with fasting insulin, HOMA-IR and Matsuda-index. Figure S5. Metabolic trajectories compared between insulin resistant individuals of normal glucose tolerance group to those with impaired fasting glucose. Figure $\mathbf{S 6}$. Metabolic trajectories for individuals with IS-NGT, IR-NGT, IFG, IGT, and NDM. Figure S7. Absolute metabolic concentrations at $0,30,60$ and 120 minute for IS-NGT, IRNGT, IFG, IGT and NDM. Figure S8. Replication in Oulu1945. Figure S9. Group comparisons adjusted with different factors. Figure S10. Difference in baseline metabolite concentration (left) and 2-h metabolic change (right) comparing the groups to the reference group (IS-NGT).

Additional file 2: Table S2. Interaction test of time groups and glucose groups for 78 metabolic measrues, adjusted for sex. Table S3. Change (\%) in relative to baseline at 30,60, 120 minute for five individual groups across 78 metabolic measures. Table S4. Differences in metabolite changes (\%) between IR-NGT and IS-NGT. Table S5. Differences in metabolite changes (\%) between IR-NGT and those with IGT or NDM.

\footnotetext{
Abbreviations

BMI: Body mass index; CVD: Cardiovascular disease; HDL: High-density lipoprotein; HOMA-IR: Homeostasis model assessment of insulin resistance; IDL: Intermediate-density lipoprotein; IFG: Impaired fasting glucose; IGT: Impaired glucose tolerance; IR: Insulin resistance; IR-NGT: Insulin resistance subgroup of normal glucose tolerance; ISI-Matsuda: Insulin sensitivity index-Matsuda; IS-NGT: Insulin-sensitive subgroup of normal glucose tolerance; LDL: Low-density lipoprotein; NDM: New onset of type 2 diabetes; NFBC66: The Northern Finland Birth Cohort 1966; NMR: Nuclear magnetic resonance; OGTT: Oral glucose tolerance test; VLDL: Very-lowdensity lipoproteins
}

\section{Acknowledgements}

Not applicable.

\section{Authors' contributions}

All listed authors meet the requirements for authorship. JJ, JA, KP, SKK, MRJ, and MAK contributed to the clinical data and study design. MAK contributed to the metabolomics. QW, JK, VPM, and MAK contributed to the analysis plan and interpretations. QW contributed to the statistical analyses. QW, VPM, and MAK drafted the manuscript. All authors commented the manuscript and agreed to its content.

\section{Funding}

This work is supported by grants from the National Health and Medical Research Council of Australia (APP1158958); the UK Medical Research Council (MC_UU_12013/1); the Academy of Finland (grant numbers 297338 and 307247); the Novo Nordisk foundation (NNF17OC0027034 and NNF170C0026062); the Health and Wellfare Center, City of Oulu, Finland; the Sigrid Juselius Foundation; the ERDF European Regional Development Fund (grant no. 539/2010 A31592); and the European Union's Horizon 2020 research and innovation programme (grant agreement no. 633595; DynaHEALTH). The Baker Institute is supported in part by the Victorian Government's Operational Infrastructure Support Program.

Availability of data and materials

Data are available for researchers who meet the criteria for access to confidential data according to the rules of each individual cohort and can be requested from the Institutional Data Access Committees of the Northern Finland Birth Cohort Study and the Oulu1945 study (University of Oulu, Finland)

Ethics approval and consent to participate

Ethical approval was obtained from the Ethics Committee of the Northern Ostrobothnia Hospital District. All participants gave written informed consent.

Consent for publication

Not applicable.

\section{Competing interests}

The authors declare that they have no competing interests.

\section{Author details}

${ }^{1}$ Computational Medicine, Faculty of Medicine, University of Oulu, Oulu, Finland. ${ }^{2}$ Systems Epidemiology, Baker Heart and Diabetes Institute, Melbourne, VIC, Australia. ${ }^{3}$ Center for Life Course Health Research, Faculty of Medicine, University of Oulu, Oulu, Finland. ${ }^{4}$ Biocenter Oulu, University of Oulu, Oulu, Finland. ${ }^{5}$ Unit of Primary Care and Medical Research Center, Oulu University Hospital, Oulu, Finland. ${ }^{6}$ Oulunkaari Health Center, li, Finland. ${ }^{7}$ NordLab Oulu, Oulu University Hospital and Department of Clinical Chemistry, University of Oulu, Oulu, Finland. ${ }^{8}$ Health and Wellfare Center, Oulu, Finland. ${ }^{9}$ Healthcare and Social Services of Selänne, Pyhäjärvi, Finland. ${ }^{10}$ Department of Epidemiology and Biostatistics, MRC-PHE Centre for Environment and Health, School of Public Health, Imperial College London, London, UK. ${ }^{11}$ Department of Life Sciences, College of Health and Life Sciences, Brunel University London, Uxbridge, Middlesex, UK. ${ }^{12}$ National Institute for Health and Welfare, Helsinki, Finland. ${ }^{13}$ Computational and Systems Biology Program, Precision Medicine Theme, South Australian Health and Medical Research Institute, Adelaide, Australia. ${ }^{14}$ Hopwood Centre for Neurobiology, Lifelong Health Theme, SAHMRI, Adelaide, Australia. ${ }^{15} \mathrm{MRC}$ Integrative Epidemiology Unit, University of Bristol, Bristol, UK. ${ }^{16}$ Population Health Science, Bristol Medical School, University of Bristol, Bristol, UK. ${ }^{17} \mathrm{NMR}$ Metabolomics Laboratory, School of Pharmacy, University of Eastern Finland, Kuopio, Finland. ${ }^{18}$ Department of Epidemiology and Preventive Medicine, School of Public Health and Preventive Medicine, Faculty of Medicine, Nursing and Health Sciences, The Alfred Hospital, Monash University, Melbourne, VIC, Australia. 
Received: 8 April 2019 Accepted: 2 October 2019

Published online: 29 November 2019

\section{References}

1. Chatterjee S, Khunti K, Davies MJ. Type 2 diabetes. Lancet. 2017;389:2239-51.

2. Emerging Risk Factors Collaboration, Sarwar N, Gao P, Seshasai SRK, Gobin R, Kaptoge $\mathrm{S}$, et al. Diabetes mellitus, fasting blood glucose concentration, and risk of vascular disease: a collaborative meta-analysis of 102 prospective studies. Lancet. 2010;375:2215-22.

3. Wang Q, Holmes MV, Davey Smith G, Ala-Korpela M. Genetic support for a causal role of insulin resistance on circulating branched-chain amino acids and inflammation. Diabetes Care. 2017:40:1779-86.

4. Samuel VT, Shulman Gl. The pathogenesis of insulin resistance: integrating signaling pathways and substrate flux. J Clin Invest. 2016;126:12-22.

5. Laakso M, Kuusisto J. Insulin resistance and hyperglycaemia in cardiovascular disease development. Nat Rev Endocrinol. 2014;10:293-302.

6. Authors/Task Force Members, Rydén L, Grant PJ, Anker SD, Berne C, Cosentino F, et al. ESC guidelines on diabetes, pre-diabetes, and cardiovascular diseases developed in collaboration with the EASD: the task force on diabetes, pre-diabetes, and cardiovascular diseases of the European Society of Cardiology (ESC) and developed in collaboration with the European Association for the Study of Diabetes (EASD). Eur Heart J. 2013;34:3035-87

7. Shaham O, Wei R, Wang TJ, Ricciardi C, Lewis GD, Vasan RS, et al. Metabolic profiling of the human response to a glucose challenge reveals distinct axes of insulin sensitivity. Mol Syst Biol. 2008:4:214.

8. Nathan DM, Davidson MB, DeFronzo RA, Heine RJ, Henry RR, Pratley R, et al. Impaired fasting glucose and impaired glucose tolerance: implications for care. Diabetes Care. 2007;30:753-9.

9. Abdul-Ghani MA, Tripathy D, DeFronzo RA. Contributions of beta-cell dysfunction and insulin resistance to the pathogenesis of impaired glucose tolerance and impaired fasting glucose. Diabetes Care. 2006;29:1130-9.

10. Guasch-Ferré M, Hruby A, Toledo E, Clish CB, Martínez-González MA, SalasSalvadó J, et al. Metabolomics in prediabetes and diabetes: a systematic review and meta-analysis. Diabetes Care. 2016:39:833-46.

11. Ruiz-Canela M, Hruby A, Clish CB, Liang L, Martínez-González MA, Hu FB. Comprehensive metabolomic profiling and incident cardiovascular disease: a systematic review. J Am Heart Assoc. 2017;6:e005705.

12. Mahendran $Y$, Vangipurapu J, Cederberg H, Stancáková A, Pihlajamäki J, Soininen $P$, et al. Association of ketone body levels with hyperglycemia and type 2 diabetes in 9,398 Finnish men. Diabetes. 2013;62:3618-26.

13. Do R, Willer CJ, Schmidt EM, Sengupta S, Gao C, Peloso GM, et al. Common variants associated with plasma triglycerides and risk for coronary artery disease. Nat Genet. 2013;45:1345-52.

14. Holmes MV, Ala-Korpela M, Smith GD. Mendelian randomization in cardiometabolic disease: challenges in evaluating causality. Nat Rev Cardiol. 2017;14:577-90.

15. Kathiresan S. Putative medicines that mimic mutations. Nature. 2017:548:530-1.

16. Vossen M, Tödter K, Altenburg C, Beisiegel U, Scheja L. Plasma triglycerides after oral glucose load specifically associate with metabolic risk markers in healthy type 2 diabetes offspring. Atherosclerosis. 2011;217:214-9.

17. Li Q, Gu W, Ma X, Liu Y, Jiang L, Feng R, et al. Amino acid and biogenic amine profile deviations in an oral glucose tolerance test: a comparison between healthy and hyperlipidaemia individuals based on targeted metabolomics. Nutrients. 2016;8:379.

18. Rämö JT, Kaye SM, Jukarainen S, Bogl LH, Hakkarainen A, Lundbom J, et al. Liver fat and insulin sensitivity define metabolite profiles during a glucose tolerance test in young adult twins. J Clin Endocrinol Metab. 2017;102:220-31.

19. Molnos S, Wahl S, Haid M, Eekhoff EMW, Pool R, Floegel A, et al. Metabolite ratios as potential biomarkers for type 2 diabetes: a DIRECT study. Diabetologia. 2017:64:718-3.

20. Ho JE, Larson MG, Vasan RS, Ghorbani A, Cheng S, Rhee EP, et al. Metabolite profiles during oral glucose challenge. Diabetes. 2013;62:2689-98.

21. Geidenstam N, Spégel P, Mulder H, Filipsson K, Ridderstråle M, Danielsson $\mathrm{APH}$. Metabolite profile deviations in an oral glucose tolerance test-a comparison between lean and obese individuals. Obesity (Silver Spring). 2014:22:2388-95

22. Järvelin M-R, Sovio U, King V, Lauren L, Xu B, McCarthy Ml, et al. Early life factors and blood pressure at age 31 years in the 1966 northern Finland birth cohort. Hypertension. 2004;44:838-46.
23. Psychogios N, Hau DD, Peng J, Guo AC, Mandal R, Bouatra S, et al. The human serum metabolome. Flower D, editor. PLoS One. 2011;6:e16957.

24. Soininen $P$, Kangas AJ, Würtz P, Tukiainen T, Tynkkynen T, Laatikainen R, et al. High-throughput serum NMR metabonomics for cost-effective holistic studies on systemic metabolism. Analyst. 2009;134:1781-5.

25. Soininen P, Kangas AJ, Würtz P, Suna T, Ala-Korpela M. Quantitative serum nuclear magnetic resonance metabolomics in cardiovascular epidemiology and genetics. Circ Cardiovasc Genet. 2015;8:192-206.

26. Würtz P, Kangas AJ, Soininen P, Lawlor DA, Davey Smith G, Ala-Korpela M. Quantitative serum nuclear magnetic resonance metabolomics in largescale epidemiology: a primer on -omic technologies. Am J Epidemiol. 2017; 186:1084-96.

27. Inouye M, Kettunen J, Soininen P, Silander K, Ripatti S, Kumpula LS, et al. Metabonomic, transcriptomic, and genomic variation of a population cohort. Mol Syst Biol. 2010;6:441.

28. Würtz $P$, Mäkinen V-P, Soininen $P$, Kangas AJ, Tukiainen $T$, Kettunen J, et al. Metabolic signatures of insulin resistance in 7,098 young adults. Diabetes. 2012;61:1372-80.

29. Würtz P, Havulinna AS, Soininen P, Tynkkynen T, Prieto-Merino D, Tillin T, et al. Metabolite profiling and cardiovascular event risk: a prospective study of three population-based cohorts. Circulation. 2015;131:774-85.

30. Kujala UM, Mäkinen V-P. Heinonen I, Soininen P, Kangas AJ, Leskinen TH et al. Long-term leisure-time physical activity and serum metabolome. Circulation. 2013:127:340-8.

31. Wang Q, Kangas AJ, Soininen P, Tiainen M, Tynkkynen T, Puukka K, et al. Sex hormone-binding globulin associations with circulating lipids and metabolites and the risk for type 2 diabetes: observational and causal effect estimates. Int J Epidemiol. 2015;44:623-37.

32. Wang TJ, Larson MG, Vasan RS, Cheng S, Rhee EP, McCabe E, et al. Metabolite profiles and the risk of developing diabetes. Nat Med. 2011;17:448-53.

33. Palmer SC, Mavridis D, Nicolucci A, Johnson DW, Tonelli M, Craig JC, et al. Comparison of clinical outcomes and adverse events associated with glucose-lowering drugs in patients with type 2 diabetes. JAMA 2016;316:313-2

34. Huang Y, Cai X, Mai W, Li M, Hu Y. Association between prediabetes and risk of cardiovascular disease and all cause mortality: systematic review and meta-analysis. BMJ. 2016;355:15953-11.

\section{Publisher's Note}

Springer Nature remains neutral with regard to jurisdictional claims in published maps and institutional affiliations.

Ready to submit your research? Choose BMC and benefit from:

- fast, convenient online submission

- thorough peer review by experienced researchers in your field

- rapid publication on acceptance

- support for research data, including large and complex data types

- gold Open Access which fosters wider collaboration and increased citations

- maximum visibility for your research: over $100 \mathrm{M}$ website views per year

At BMC, research is always in progress.

Learn more biomedcentral.com/submissions 